\title{
A Coupled Resonator for Highly Tunable and Amplified Mixer/Filter
}

\author{
Saad Ilyas, Nizar Jaber, and Mohammad I. Younis
}

\begin{abstract}
We present an H-shaped resonator made of two clamped-clamped microbeams mechanically coupled at the middle with a strong coupler to achieve, in a single device, mechanical amplification of the response signal, filtering and frequency conversion simultaneously. Using mechanical amplification combined with combination resonances generated from a mixed-frequency excitation, a wideband tunable filter and a simultaneous frequency up and down convertor at multiple bands is demonstrated. The proposed coupled structure, when combined with the easy-to-implement technique of frequency mixing, is promising for applications in an RF chain.
\end{abstract}

Index Terms-mechanically coupled resonator, mixedfrequency excitation, mixer/filter, response amplification.

\section{INTRODUCTION}

$\mathrm{W}$ ITH the advances in Micro/Nano electromechanical MEMS/NEMS fabrication and the effort to miniaturize resonant sensors/actuators into the nano regime, the transduction of these devices for electronic readout becomes challenging. Significant efforts have been dedicated to improve the signal-to-noise ratio by mechanical amplification of the signal prior to the electronic amplification. Most of the explored methods in mechanically coupled resonators have relied mainly on parametric amplification, in which the mechanical amplification is achieved by exciting the pump beams at twice the resonance frequency (pump frequency) [17]. As a result, the small signal generated by one beam is amplified at the other beam through a weak coupler [1-3, 6].

Significant efforts have been directed towards developing high-Q high-frequency devices for applications in wireless communications. These include thin film bulk wave acoustic resonators [8], aluminum nitride (AIN) based rectangular plate and ring-shaped contour-mode resonators [9], and recently an AIN Lamé mode resonators [10]. Furthermore, mechanically and electrostatically coupled structures have been successfully used in such communication applications [10-18]. Considerable effort has been directed towards achieving a highly selective tunable filter, where the bandwidth and the center frequency can be tuned efficiently [19-23]. Moreover, recent research has been dedicated to achieve filtering and frequency up/down conversion on a single device to broaden

S. Ilyas, N. Jaber, and M. I. Younis are with Physical Sciences Division, King Abdullah University of Science and Technology, Thuwal 23955-6900, KSA. This research has been sponsored through King Abdullah University of Science and Technology research funds. (emails: saad.ilyas@kaust.edu.sa, nizar.jaber@kaust.edu.sa, mohammad.younis@kaust.edu.sa) the limits of miniaturization and optimize power consumption in portable communication devices [24, 25]. Signal amplification in such devices can provide significant advantages, for example in bandpass filters, where an amplified response results in a sharper distinct pass band compared to noise, and in frequency converters, where an amplified low-loss converted signal is desired.

In this work we present a resonator, of an " $\mathrm{H}$ " shape, made of two clamped-clamped (C-C) beams coupled strongly at their midpoint using a beam coupler of almost the same size as an individual $\mathrm{C}-\mathrm{C}$ beam. The coupler is attached at the maximum displacement location of the beams. By exploiting the dynamic response of this coupled structure, maximum signal amplification (mechanical transformation) is achieved. Furthermore, we excite the structure using mixed-frequency electrostatic forcing [26-28] to achieve a wide relatively flat and amplified pass-band filtering and simultaneous mixing characteristics using a single resonator body.

\section{RESULTS AND DISCUSSION}

Fig. 1 shows (a) a schematic, (b) an SEM image, and (c) a cross-sectional view of the physical layers comprising the structure. The " $H$ " resonator is fabricated using a surface micromachining process with polyimide as the structural layer [29]. The electrode network is laid down such that it allows different options to actuate the resonator and detect its response. The device can be actuated through either of the $\mathrm{C}-\mathrm{C}$ beams: right beam (RB), left beam (LB), and through the middle coupling element (MC). Similarly, the response can be measured through any of the three beams.

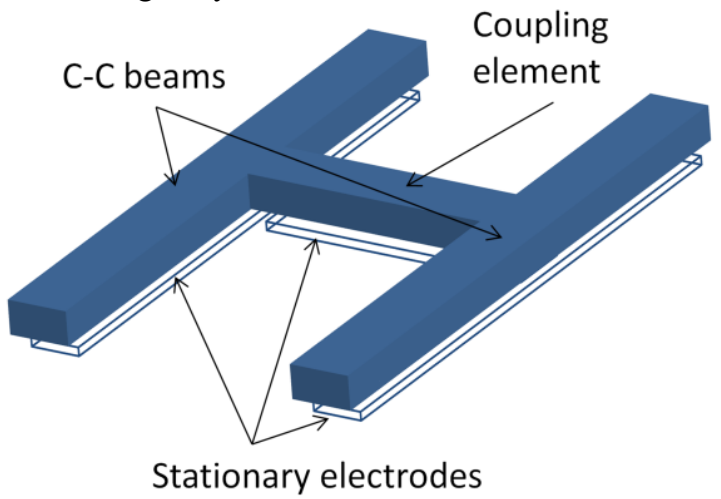

(a) 


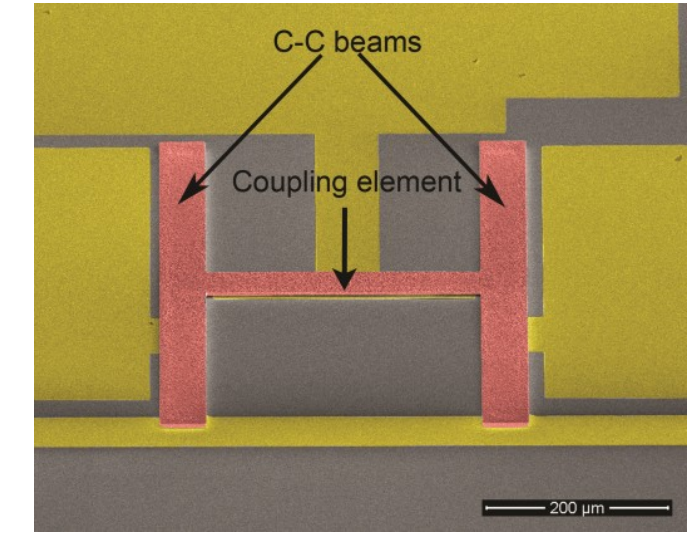

(b)

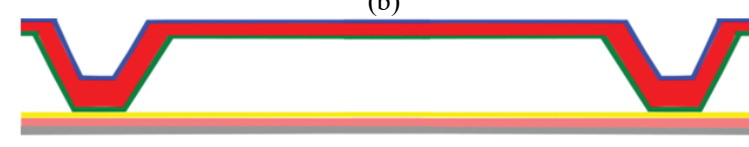

Si Substrate SiO $\mathrm{Cr} / \mathrm{Au} \quad \mathrm{Cr} / \mathrm{Au} / \mathrm{Cr}$ Polyimide $\mathrm{Ni}$

(c)

Fig. 1. (a) Schematic of the $\mathrm{H}$ resonator. (b) Colorized SEM image of the $\mathrm{H}$ resonator. (c) The cross-section of the $\mathrm{H}$ resonator showing the various fabricated layers. The two identical clamped-clamped beams are $300 \mu \mathrm{m}$ long and $50 \mu \mathrm{m}$ wide while the coupling beam is $300 \mu \mathrm{m}$ long and $35 \mu \mathrm{m}$ wide. The thickness of the $\mathrm{H}$ resonator is $5.2 \mu \mathrm{m}$ with an operating gap of $2 \mu \mathrm{m}$.

A high frequency laser Doppler Vibrometer, MSA-500 Polytec Micro System Analyzer, is used for dynamic measurements, Fig. 2(a). The resonator under test is placed inside a vacuum chamber positioned directly underneath the lens of the Vibrometer. To excite the resonator, an electrostatic input signal generated in Labview is applied through a data acquisition card (DAQ) NI 6251 from National Instruments, to the right $\mathrm{C}-\mathrm{C}$ beam between the movable electrode and the bottom fixed electrode. The out-of-plane displacement data measured by the Vibrometer is post processed through the DAQ and collected at the output. The experiments are performed under low vacuum of a pressure of 1.8-3.5 mtorr. The proposed resonator is excited from the c-c beam and the response is sensed at the middle coupler, hence having different sensing and actuation areas. The electromechanical coupling coefficient $\eta_{e}=\frac{\varepsilon A_{1} V_{D C}}{g^{2}}$, defined as the ratio between the applied excitation force and the input signal is calculated to be $3.31 \times 10^{-7} \mathrm{C} / \mathrm{m}$, where $A_{1}$ is the overlap (actuation) area of the c-c beam, $\varepsilon$ is the relative permittivity, and $g$ is the gap [30]. Similarly, the read-out coupling coefficient $\eta_{r}=\frac{\varepsilon A_{2} V_{D C}}{g^{2}}$, which is the ratio between the output current and the displacement of the beam, is calculated to be $2.317 \times 10^{-7} \mathrm{C} / \mathrm{m}$, where $A_{2}$ is the overlap (sensing) area of the coupling element. Different sensing and actuation areas allow us to improve either of the coupling coefficients independently by modifying their dimensions. However, it must be noted that the $\mathrm{H}$ resonator will give the best mechanical amplification, for exact same dimensions of the beams and the coupling element, where the coupling coefficient will be same for both cases.

Figs. 2 (b)-(d) show the response of the coupled resonator. The electrostatic input is applied to the RB only, while the output signal is received, in the form of the resonator's maximum amplitude of vibration, at the midpoint of the coupling beam and C-C beam. It can be clearly observed that the output signal received at the midpoint of the coupling beam, Fig. 2(c), is almost 4 times amplified compared to the

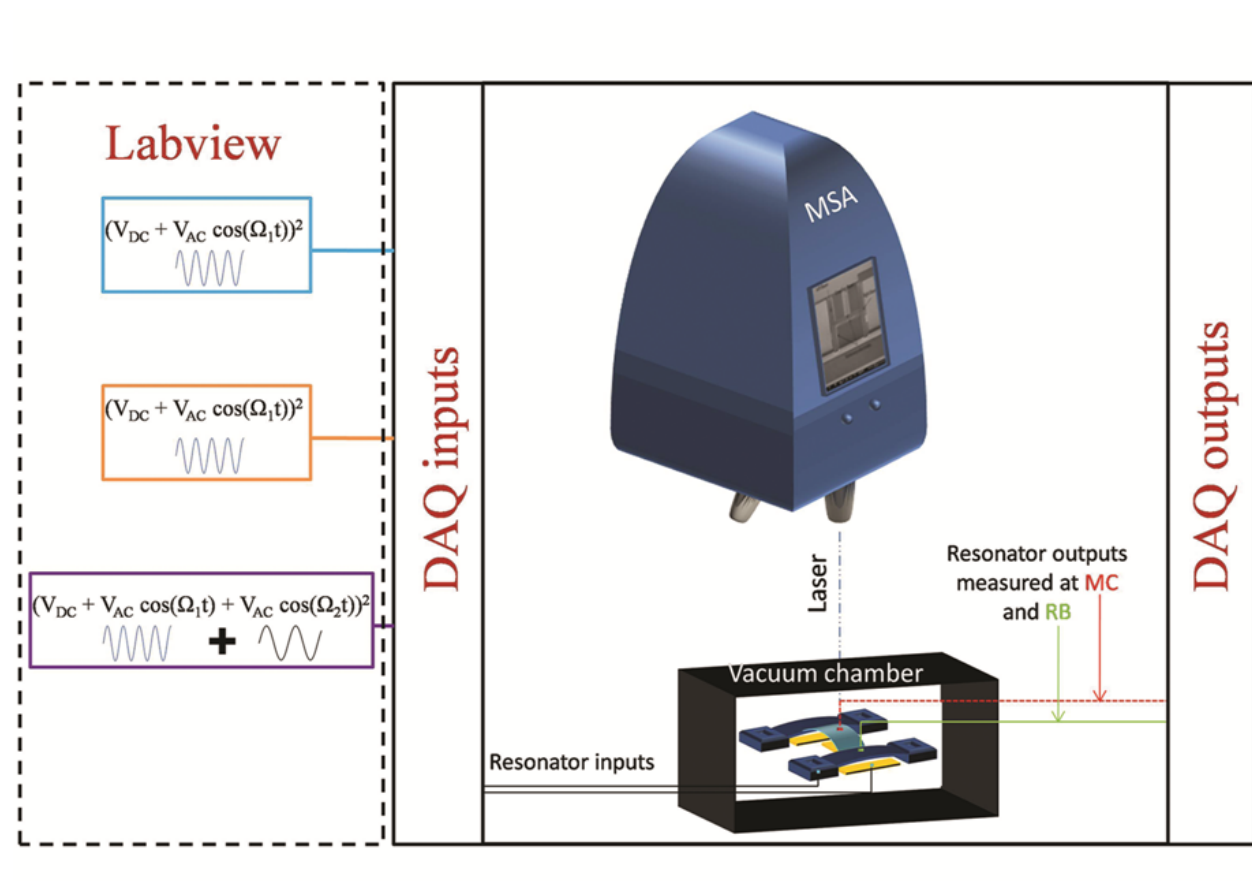

(a)

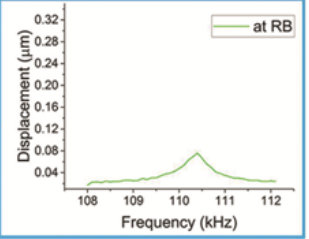

(b)

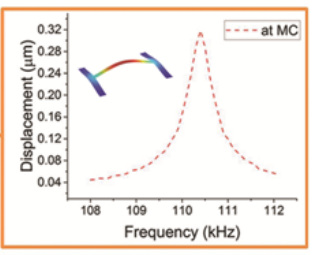

(c)

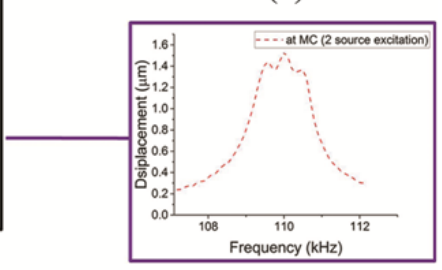

(d)

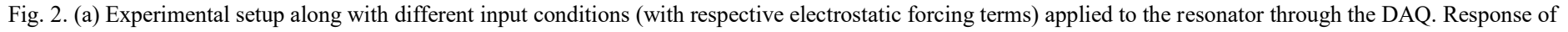
the $\mathrm{H}$ resonator under an electrostatic actuation of $\mathrm{V}_{\mathrm{DC}}=2 \mathrm{~V}$ and $\mathrm{V}_{\mathrm{AC}}=5 \mathrm{~V}$ measured at (b) midpoint of $\mathrm{RB}$ and (c) midpoint of $\mathrm{MB}$ in its first fundamental mode of

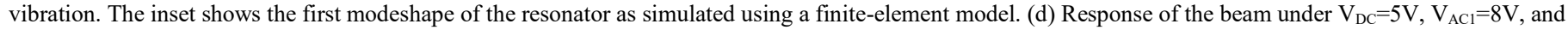

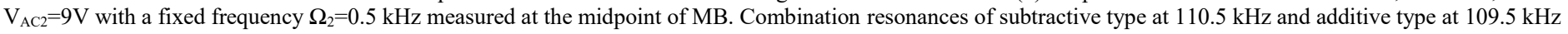

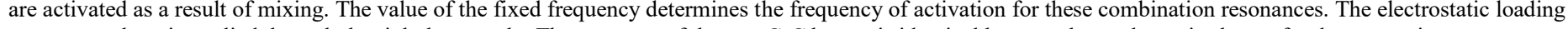
here is applied through the right beam only. The response of the two $\mathrm{C}-\mathrm{C}$ beams is identical hence only one beam is chosen for demonstration. 
output signal received at any of the $\mathrm{C}-\mathrm{C}$ beams, Fig. 2(b). It has been shown [31] that the maximum amplitude of the coupler is achieved when the base motion has close frequency to that of the coupler, i.e., both of the $\mathrm{C}-\mathrm{C}$ beams and the coupling beams have almost the same dimensions. Hence the coupled system here can be viewed as a base excitation [32] case, where the two C-C beams supply the base motion to the coupling beam sitting on top. We notice that by exciting any of the $\mathrm{C}-\mathrm{C}$ beams of the $\mathrm{H}$ resonator we receive the same level response at the two beams and an amplified response at the coupling beam. It is important to mention here that this device behaves as a mechanical amplifier, where it transforms small dynamic response amplitude at the clamped-clamped beam to high response amplitude at the middle coupling element. This should not be confused with conventional electrical amplifiers available commercially. This mechanical amplification provided by such structure holds great potential in mixing and filtering applications.

To achieve mixing and to realize an amplified response at certain band of frequency, we exploit the quadratic electrostatic force and excite the $\mathrm{H}$ resonator with two or more excitation sources. This triggers the so-called combination resonances [26-28]. In the case of two AC harmonic sources in addition to the DC load, the electrostatic excitation load can be expanded as

$\left[V_{D C}+V_{A C 1} \cos \left(\Omega_{1} t\right)+V_{A C 2} \cos \left(\Omega_{2} t\right)\right]^{2}=V_{D C}{ }^{2}+V_{A C 1}{ }^{2} \cdot \cos ^{2}\left(\Omega_{1} t\right)+V_{A C 2}{ }^{2} \cdot \cos ^{2}\left(\Omega_{2} t\right)+$

$2 V_{D C} V_{A C 1} \cdot \cos \left(\Omega_{1} t\right)+2 V_{D C} \cdot V_{A C 2} \cdot \cos \left(\Omega_{2} t\right)+V_{A C 1} V_{A C 2} \cdot\left[\cos \left\{\left(\Omega_{1}-\Omega_{2}\right) t\right\}+\cos \left\{\left(\Omega_{1}+\Omega_{2}\right) t\right\}\right]$

One can note from the last two terms of Equation (1) that whenever the sum or the difference of the excitation frequencies $(\Omega 1$ and $\Omega 2)$ equals the resonance frequency of the resonator, the resonator will experience resonance and respond with high amplitude. Fig. 2(d) shows the response of the $\mathrm{H}$ resonator in response to a similar excitation shown in Fig 2(a), measured at the midpoint of the coupling beam. The activation of combination resonances in the neighborhood of the fundamental resonance frequency is observed. Adjusting the number, values, and associated $\mathrm{V}_{\mathrm{AC}}$ of added frequencies, specific response of the resonator can be produced, which when combined with the mechanical amplification from the coupled structure can be used for simultaneous amplification, mixing and filtering applications.

Toward this, we first show in Fig. 3 the response of the resonator under multiple sources of excitation. The electrostatic loading applied to the beam is adjusted in each case to reduce the ripples and produce a relatively flat pass band response. The response of the resonator to a single source excitation (blue squares) compared against the response of the resonator for two (black solid), three (red solid circles), and four (green empty circles) AC frequency sources. Table 1 summarizes the center frequency $\left(f_{c}\right)$ and the percent bandwidth (BW) for the resonator for various excitation schemes.

It is observed that by choosing the fixed frequencies very small, the combination resonances are activated closer to the first resonance frequency of the $\mathrm{H}$ resonator, thereby increasing its bandwidth. We notice that for every new mixed- frequency, two combination resonances are activated [26-28]. As the number of mixed frequencies is increased, the bandwidth increases. Hence, one can achieve a wide-band filter by increasing the number of mixed frequencies. Although the addition of combination resonances widens the band, does not affect the order of the filter. These resonances are generated as a result of the excitation scheme and not due to the addition of more resonators to the coupled array. Hence, the filter will stay as a $2^{\text {nd }}$ order no matter how many additional combination resonances are activated via frequency mixing techniques. Each of the combination resonances can be adjusted in amplitude by controlling the input voltages applied to the system. This is an effective way to resolve the commonly encountered frequency mismatch problem in MEMS filters, which is otherwise solved by using complex structures and techniques.

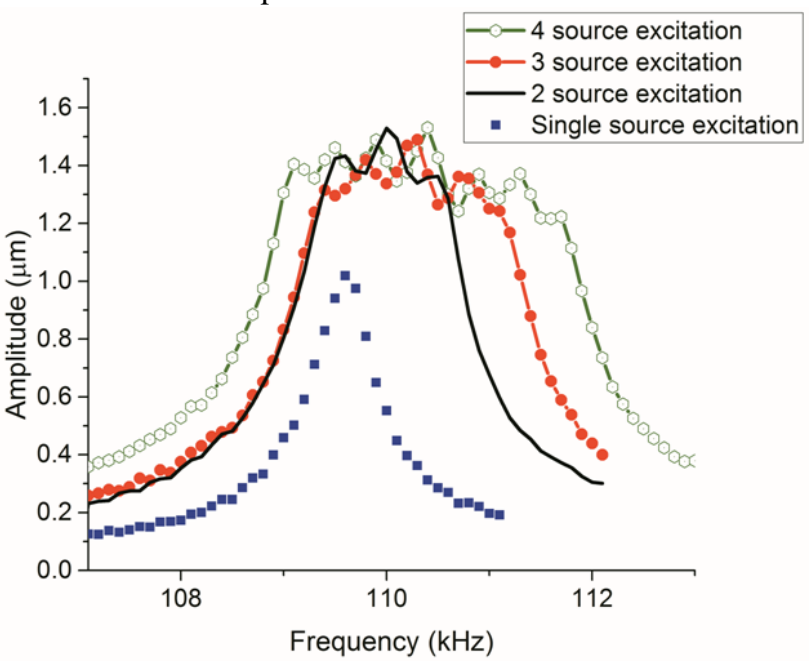

Fig. 3. Response of the H resonator under mixed-frequency excitation. Different voltages are applied for each case to produce a wide pass band response. A load of $\mathrm{V}_{\mathrm{DC}}=5 \mathrm{~V}$ and $\mathrm{V}_{\mathrm{AC}}=10 \mathrm{~V}$ is used for single source excitation (blue squares). The quality factor of the resonator is measured to be $\sim 211$. A load of $\mathrm{V}_{\mathrm{DC}}=5 \mathrm{~V}, \mathrm{~V}_{\mathrm{AC} 1}=8 \mathrm{~V}$, and $\mathrm{V}_{\mathrm{AC} 2}=9 \mathrm{~V}$ with a fixed frequency $\Omega_{2}=0.5 \mathrm{kHz}$ is used for two source excitation (solid black line). A load of $\mathrm{V}_{\mathrm{DC}}=5 \mathrm{~V}$,

$\mathrm{V}_{\mathrm{AC} 1}=6 \mathrm{~V}, \mathrm{~V}_{\mathrm{AC} 2}=6 \mathrm{~V}$, and $\mathrm{V}_{\mathrm{AC} 3}=9 \mathrm{~V}$ with fixed frequencies $\Omega_{2}=0.5 \mathrm{kHz}$ and $\Omega_{3}=0.9 \mathrm{kHz}$ is used for the three source excitation (solid red circles). A load of $\mathrm{V}_{\mathrm{DC}}=5 \mathrm{~V}, \mathrm{~V}_{\mathrm{AC} 1}=6 \mathrm{~V}, \mathrm{~V}_{\mathrm{AC} 2}=6 \mathrm{~V}, \mathrm{~V}_{\mathrm{AC} 3}=9 \mathrm{~V}$, and $\mathrm{V}_{\mathrm{AC} 4}=9 \mathrm{~V}$ with fixed frequencies $\Omega_{2}=0.5 \mathrm{kHz}, \Omega_{3}=0.9 \mathrm{kHz}$, and $\Omega_{4}=1.3 \mathrm{kHz}$ is used for the four source excitation (empty green circles). The electrostatic loading is applied through the right beam only while the response amplitude of the beam is measured at the midpoint of each of the coupling beam.

TABLE I

SUMMARY OF FILTER PARAMETERS FOR VARIOUS EXCITATION SCHEMES.

\begin{tabular}{cc|c} 
Excitation Scheme & $f_{c}(\mathrm{kHz})$ & $\% \mathrm{BW}$ \\
Single source & 109.6 & 0.5 \\
2 sources & 110 & 1.31 \\
3 sources & 110.3 & 1.84 \\
4 sources & 110.4 & 2.67 \\
\hline \hline
\end{tabular}

It is important to note here that the value and the associated $\mathrm{V}_{\mathrm{AC}}$ of the mixing frequency sources directly impact the flatness and bandwidth of the filter. The AC voltages associated with the mixing frequencies control the amplitude of the activated combination resonances while the values of mixing frequencies set the frequency of excitation of 
combination resonances [26-28]. The fixed frequency values and associated $\mathrm{AC}$ voltages are best chosen in such a way that the combination resonances are excited right next to each other with almost equal amplitudes. These resonances should not overlap each other as they will reduce the maximum achievable bandwidth and also should not be far from each other as it will adversely affect the flatness by increasing the ripples. There is always a tradeoff between the bandwidth and the flatness of the response for a given excitation scheme. A very flat response will result in less bandwidth while a wider band response will affect the flatness poorly for a given excitation scheme. For example, a 2 source excitation can provide a wide band response equal to that of 4 source excitation (by choosing the right frequency values); however the level of flatness in the case of 2 source excitation will be much worse compared to 4 source excitation.

Another desirable feature is to tune the center frequency of the resonator in real-time and while in-use using the same structure. This can also be achieved by biasing the coupling beam separately with a DC source in addition to the actuation through the right beam of the $\mathrm{H}$ resonator.

Fig. 4 shows the response of the $\mathrm{H}$ resonator under various DC biasing for the case of two source mixing. The tuning $(\Delta f)$ as a result of the DC bias to the middle coupler will stay constant regardless of the excitation scheme used, hence only one case is shown here as an example. It can be noticed that the band of the filter can be tuned without affecting the bandwidth or any other parameters. The overall amplitude of the filter decreases slightly as the overall structure becomes stiffer, and hence it needs more power to oscillate at similar levels of amplitude. Fig. 4 also indicates a positive frequency shift in the resonator due to the induced tension in the coupler from the DC bias.

Next, we demonstrate simultaneous frequency up and down conversion at multiple bands. Since the resonator always vibrates with the resonance frequency at these combination resonances, we utilize this property of the mixed-frequency excitation for frequency conversion [26-28]. Fig. 5 shows the response of the resonator under mixed frequency excitation. Depending on the selection of the fixed frequency we are able to generate two wide bands of the frequencies far apart from the resonance frequency of the $\mathrm{H}$ resonator. Any frequency at these bands is converted into the resonance frequency of the resonator, hence achieving the up conversion for the additive type resonances and down conversion for the subtractive type combination resonances. Using such coupled structure provides the benefit of an amplified response after the frequency conversion, which is desired. Furthermore, depending on the number and values of frequencies used for mixing, several bands of frequencies can be generated and can perform the frequency up/down conversion simultaneously while amplifying the response.

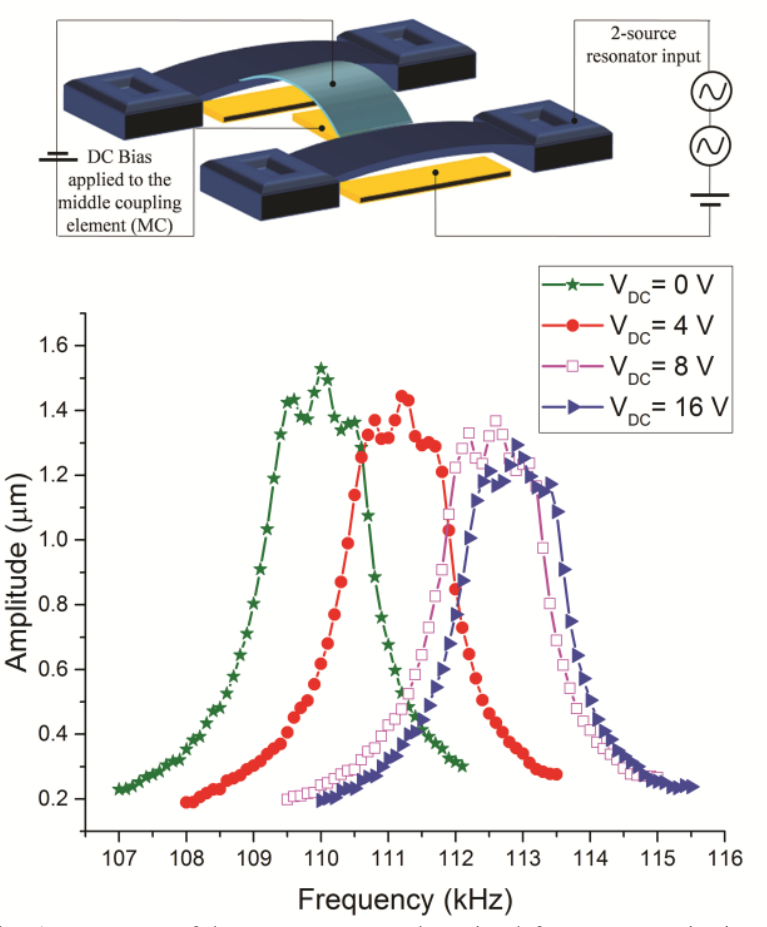

Fig. 4. Response of the $\mathrm{H}$ resonator under mixed-frequency excitation of $\mathrm{V}_{\mathrm{DC}}=5 \mathrm{~V}, \mathrm{~V}_{\mathrm{ACl}}=8 \mathrm{~V}$, and $\mathrm{V}_{\mathrm{AC} 2}=9 \mathrm{~V}$ with a fixed frequency $\Omega_{2}=0.5 \mathrm{kHz}$ applied through the right beam. The coupling beam is biased separately to change the stiffness of the structure to tune the center frequency of the resonator.

One important point to address here is that the overall power consumption of such a device can be high, when compared to the state of the art coupled structures for filtering applications, due to the use of additional sources. Such a structure however provides advantages in term of integration densities and also reduces some of the excess power consumption, as it can provide several functionalities using a single resonator. Similarly using a single structure also helps solving various mismatch problems that arise due to using separate elements for each of amplification, filtering, and mixing in an RF chain.

Flexural mode MEMS resonators in general have high motional resistances ranging from $\mathrm{k} \Omega$ to $\mathrm{M} \Omega$, which causes impedance mismatch issues when used in an RF electronic chain. In general, this can be dealt with by using proper termination resistances with the aid of appropriate CMOS electronics [22] to match the impedance with the load, and hence achieve maximum power transfer. This however adds complexity to the circuit. The motional resistance can be defined as, $R_{m}=\frac{\sqrt{k \cdot m}}{Q \eta^{2}}=\frac{k \cdot g^{4}}{Q \varepsilon^{2} \omega_{n} A^{2} V_{D C}^{2}}$, where $R_{m}$ is directly proportional to the stiffness $k$, and the fourth power of the operation gap $g$, while it is inversely proportional to the quality factor $Q$, natural resonant frequency $\omega_{n}$, overlap area $A$, and the applied $V_{D C}$. We also notice that most of these variables depend on each other, and hence, there exist optimized design parameters that should be reached to achieve the desired motional resistance for maximum transfer of power. Reducing the gap will have the most prominent effect. However there are limitations to how small a gap can be considering fabrication limitations and permanent collapse of 
the device to the stationary electrode. Hence, the gap, the overlap area, and bias voltage need to be optimized such that the overall $R_{m}$ value is reduced. Similarly, changing the fabrication material and minimizing the squeeze film damping of the resonator, such that the quality factor is increased, will result in reducing the overall $R_{m}$ value. Adjusting these parameters will set the impedance value that maximizes power transfer.

In summary, we presented a technique to amplify (transform) a mechanical response using an $\mathrm{H}$ shaped mechanically coupled resonator. We demonstrated the ability of the coupler to provide a tunable, amplified filtering operation using mixed-frequency excitation. In addition, we also demonstrated the ability of the structure to perform simultaneous frequency up/down conversions at several bands with an amplified response. The device in its current design has limited applications due to its low center frequency in the low frequency regime, such as in biomedical applications, navigation systems, and low frequency RF identification applications, where the frequencies of operation range from a few hertz to hundreds of $\mathrm{kHz}$ [33-35]. However, with a more dedicated design based on a stiffer material and of smaller dimensions, high frequency applications can be targeted. It is worth to mention that the device presented here is a proof-ofconcept demonstrating using a single strongly coupled $\mathrm{H}$ resonator under mixed frequency excitation to perform multiple operations required in an RF chain. The performance parameters, i.e., center frequency, quality factor, and tuning properties, of the device can be further improved with more optimized design. For instance, the centre frequency of the device can be significantly increased by reducing the size of the proposed resonator. However, scaling down to nano scale can adversely affect other parameters of the filter like insertion losses. Another key factor to improve the center frequency of the device is the fabrication material. Note here that the presented prototype resonator is made of polyimide, which is very compliant material, hence having low natural frequencies. Changing the material to Silicon only can yield very high center frequencies and quality factors.

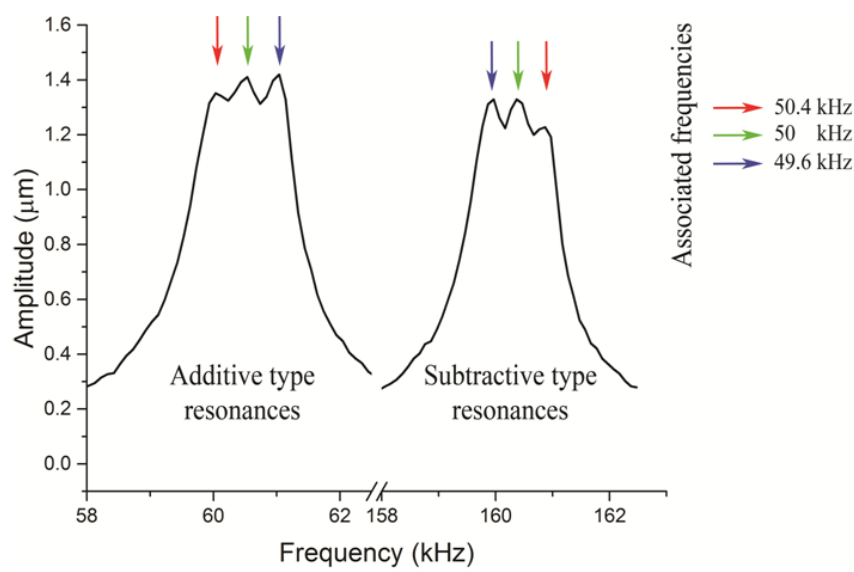

Fig. 5. Response of the $\mathrm{H}$ resonator under four source excitation with loading of $\mathrm{V}_{\mathrm{DC}}=4 \mathrm{~V}, \mathrm{~V}_{\mathrm{AC} 1}=6 \mathrm{~V}, \mathrm{~V}_{\mathrm{AC} 2}=6 \mathrm{~V}, \mathrm{~V}_{\mathrm{AC} 3}=9 \mathrm{~V}$, and $\mathrm{V}_{\mathrm{AC} 4}=9 \mathrm{~V}$ at fixed frequencies $\Omega_{2}=49.6 \mathrm{kHz}, \Omega_{3}=50 \mathrm{kHz}$, and $\Omega_{4}=50.4 \mathrm{kHz}$ applied through the right beam.

Each of the associated fixed frequency is responsible for activating simultaneously an additive and subtractive resonance each.

Having these features in a single structure has promising potential in wireless communication applications, where added power consumption is avoided to run separate devices for filtering/frequency conversion, thereby overcoming the limitation of frequency mismatch problems between mixer output and filter input causing additional insertion losses and provides a compact design for portable communication devices.

\section{REFERENCES}

[1] R. B. Karabalin, X. L. Feng, and M. L. Roukes. "Parametric nanomechanical amplification at very high frequency." Nano Lett., vol. 9, no. 9, pp. 3116-3123, 2009.

[2] S.B. Shim, M. Imboden, and, P. Mohanty., Synchronized oscillation in coupled nanomechanical oscillators. Science, vol. 316, no. 5821, pp. 9599, 2007.

[3] Eichler, J. Chaste, J. Moser, and A. Bachtold. "Parametric amplification and self-oscillation in a nanotube mechanical resonator." Nan. Lett., vol. 11, no. 7, pp. 2699-2703, 2011.

[4] D. W. Carr, S. Evoy, L. Sekaric, H. G. Craighead, and J. M. Parpia. "Parametric amplification in a torsional microresonator." App. Phy. Lett., vol. 77, no. 10, pp. 1545-1547, 2000.

[5] J. T. M. Van Beek, K. L. Phan, G. J. A. M. Verheijden, G. E. J. Koops, C. Van der Avoort, J. Van Wingerden, D. ErnurBadaroglu, J. J. M. Bontemps, and R. Puers. "A piezo-resistive resonant MEMS amplifier." IEEE Int. Electron Dev. Meet.10.1109/IEDM.2008.4796782,2008.

[6] R. Baskaran, and K. L. Turner. "Mechanical domain coupled mode parametric resonance and amplification in a torsional mode micro electro mechanical oscillator." J. Micromech. Microeng.,vol. 13, no. 5, pp. 701,2003

[7] K. L. Turner, S. A. Miller, P. G. Hartwell, N. C. MacDonald, S. H. Strogatz, and S. G. Adams. "Five parametric resonances in a microelectromechanical system." Nature, vol. 396, no. 6707, pp. 149152, 1998.

[8] G. Piazza, P. J. Stephanou, and A. P. Pisano. "Piezoelectric aluminum nitride vibrating contour-mode MEMS resonators." J.Microelectromech. Syst. vol. 15, no. 6, pp. 1406-1418, 2006.

[9] R. C. Ruby, P. Bradley, Y. Oshmyansky, A. Chien, and J. D. Larson. "Thin film bulk wave acoustic resonators (FBAR) for wireless applications." In Ultrasonics Symposium, 2001 IEEE, vol. 1, pp. 813821. IEEE, 2001.

[10] C. Cassella, Y. Hui, Z. Qian, G. Hummel, and M. Rinaldi. "Aluminum Nitride Cross-Sectional Lamé Mode Resonators." J.Microelectromech. Sys. vol. 25, no. 2, pp. 275-285, 2016.

[11] M. M. Shalaby, M. A. Abdel moneum, and K. Saitou, "Design of spring coupling for high-Q high-frequency MEMS filters for wireless application," IEEE Trans. Ind. Electron., vol. 56, no. 4, pp. 1022-1030, Apr. 2009.

[12] F. D. Bannon, III, J. R. Clark, and C. T.-C. Nguyen, "High- $Q H F$ micromechanical filters", IEEE J. Solid-State Circuits, vol. 35, pp.512 $526,2000$.

[13] S. Pourkamali, and F. Ayazi. "Electrically coupled MEMS bandpass filters: Part I: With coupling element." Sensor. Actuat. A-Phys, vol. 122, pp. 307-316, 2005.

[14] S. Pourkamali, and F. Ayazi. "Electrically coupled MEMS bandpass filters: Part II. Without coupling element." Sensor. Actuat. A-Phys, vol. 122, pp. 317-325, 2005.

[15] M.-H. Li, C.-Y. Chen, W.-C. Chen, and S.-S. Li, "A vertically coupled MEMS resonator pair for oscillator applications," J. Microelectromech. Syst., vol. 24, no. 3, pp. 528-530, Jun. 2015. DOI: 10.1109/JMEMS.2015.2421555.

[16] Y. Chen, M. H. Li, C. H. Chin, C. S. Li, and S. S. Li, "Combined electrical and mechanical coupling for mode-reconfigurable CMOSMEMS filters." Proc. 27th IEEE Int. Conf. Micro Electro Mech. Syst, 
pp. 1249-1252, Jan, 2014.

[17] B. K. Hammad, "Natural frequencies and mode shapes of mechanically coupled microbeam resonators with an application to micromechanical filters," Shock. Vib., vol. 2014, 2014.

[18] R. Abdolvand, G.K. Ho, and F. Ayazi, "Poly-wire-coupled single crystal silicon HARPSS micromechanical filters using oxide islands". Tech. Dig. Hilton Head, pp.242-245, 2004.

[19] Y. S. Lin, Y. Qian, F. Ma, Liu Z, P. Kropelnicki, C. Lee. "Development of stress-induced curved actuators for a tunable $\mathrm{THz}$ filter based on double split-ring resonators". Appl. Phy. Lett. vol. 102, no. 11, pp. 111908, 2013.

[20] M. S. Hajhashemi, A. Amini, B. Bahreyni. "A micromechanical bandpass filter with adjustable bandwidth and bidirectional control of centre frequency". Sensor. Actuat. A-Phys, vol. 187, pp. 10-15, 2012.

[21] T. L. Naing, J. N. Nilchi, R. Liu, T. O. Rocheleau, C. T. Nguyen. "Active Q-control for improved insertion loss micromechanical filters". IEEE Frequency Control Symposium (FCS), pp. 1-6. May, 2015.

[22] J. L. Lopez, J. Verd, A. Uranga, J. Giner, G. Murillo, F. Torres, G. Abadal, N. Barniol. "A CMOS-MEMS rf-tunable bandpass filter based on two high-22-mhz polysilicon clamped-clamped beam resonators". Electron Dev. Lett., vol. 30, no. 7, pp. 718-720, 2009.

[23] X. Zou, J. Yan, A. A. Seshia. "A novel tuneable band-pass filter based on a single square-ring MEMS resonator". Procedia Engineering.vol. 31, no. 5, pp. 1446-1449, 2010.

[24] A. C. Wong, C. T. Nguyen. "Micromechanical mixer-filters (" mixlers")”. J. Microelectromech. Syst., vol. 13, no. 1, pp. 100-112, Jun. 2004.

[25] F. Chen, J. Brotz, U. Arslan, C. C. Lo, T. Mukherjee, G. K. Fedder. "CMOS-MEMS resonant RF mixer-filters", .Proc. 18th IEEE Int. Conf. Micro Electro Mech. Syst, pp. 24-27, Jan, 2005.

[26] S. Ilyas, A. Ramini, A. Arevalo, and M.Younis, “An Experimental and Theoretical Investigation of a Micromirror UnderMixed-Frequency Excitation.", J. Microelectromech. Syst., vol. 24, no. 4, pp. 1124 -1131, 2015.

[27] N. Jaber, A. Ramini, and M. I. Younis, "Multifrequency excitation of a clamped-clamped microbeam: Analytical and experimental investigation," Microsystems \& Nanoengineering, vol. 2, 2016.

[28] N. Jaber, A. Ramini, Q. Hennawi, and M. I. Younis, "Wideband MEMS resonator using multifrequency excitation," Sensors and Actuators A: Physical, vol. 242, pp. 140-145, 2016.

[29] A. Arevalo, E. Byas, D.Conchouso, D. Castro, S. Ilyas, I. G. Foulds, “A Versatile Multi-User Polyimide Surface Micromachining Process for MEMS Applications". IEEE Int. Con. Nano/Micro Engineered and Molecular Systems NEMS2015, Xia'an., pp. 561-565, 10.1109/NEMS.2015.7147492, 7-11 April, 2005.

[30] L. Méndez, J. Luis, and B. I. Beumala. "Application of CMOS-MEMS integrated resonators to RF communicaction systems". Universitat Autònoma de Barcelona, 2009.

[31] S. Ilyas, N. Jaber, and M. I. Younis. "Static and Dynamic Amplification Using Strong Mechanical Coupling." J. Microelectromech. Syst.vol. 25, no. 5, pp. 916-921, 2016.

[32] M. I. Younis, MEMS Linear and Nonlinear Statics and Dynamics. (Springer, 2011).

[33] B.K. Saurabh, Y.N. Kumar, S. Patidar, and M.H.Vasantha, "Design and Implementation of Tunable Bandpass Filter for Biomedical Applications." IEEE Nanoelectronic and Information Systems (iNIS), pp. 43-46, 2016.

[34] C.M. Roberts, "Radio frequency identification (RFID)". Computers \& security, vol. 25, no. 1, pp.18-26, 2006.

[35] JB. Hagen, "Radio-frequency electronics: circuits and applications." Cambridge University Press, 2009. 